\title{
Endoscopic Optic Nerve Decompression Through Supraorbital Keyhole Extradural Approach: A Cadaveric Study
}

\author{
Xiaodong WANG ${ }^{1,2^{*}}$, Wei WU ${ }^{2 *}$, Hengzhu ZHANG ${ }^{2}$, Qing LAN ${ }^{1}$ \\ ${ }^{1}$ The Second Affiliated Hospital of Soochow University, Department of Neurosurgery, Suzhou, Jiangsu Province, China \\ ${ }^{2}$ Clinical Medical College of Yangzhou University, Department of Neurosurgery, Yangzhou, Jiangsu Province, China \\ *Xiaodong WANG and Wei WU contributed equally to this article, so they are common first authors.
}

\section{ABSTRACT}

AIM: Many approaches for optic nerve decompression have their own advantages and disadvantages. The aim of this study was to perform endoscopic decompression of optic nerve through the supraorbital keyhole and evaluate its feasibility.

MATERIAL and METHODS: Ten adult cadaveric heads were studied using 4-mm, 0- and 30-degree rigid endoscopes to perform the optic nerve decompression through the supraorbital keyhole extradural approach. Furthermore, the relevant measurements about the optic canal were recorded.

RESULTS: Through the supraorbital keyhole, the endoscope was introduced into the extradural space, and the following structures could be exposed and identified: the sphenoid ridge, the anterior clinoid process, the roof of the optic canal and the falciform ligament. The roof and lateral wall of the optic canal were removed using a drill under the endoscope, and more sufficient decompression could be achieved by further incising the falciform ligament and optic sheath. After measurement, the distance from the zygomatic process of the frontal bone to the optic canal was $59.32 \pm 2.27 \mathrm{~mm}$, the distance from the upper midpoint of the posterior foramen of the optic canal to the internal carotid artery was $3.80 \pm 0.93 \mathrm{~mm}$.

CONCLUSION: According to the cadaveric study, it is feasible to perform the endoscopic decompression of optic nerve with a clear view through the supraorbital keyhole extradural approach. Our study may provide a minimally invasive and safe option for the optic nerve decompression.

KEYWORDS: Supraorbital keyhole, Endoscope, Optic nerve decompression

\section{INTRODUCTION}

$\mathrm{M}$ any surgical approaches have been described for decompression of the optic nerve to treat traumatic optic neuropathy $(14,18,21,22)$. The most commonly used approaches include the transcranial and the transnasal approaches (15), and each of them has specific advantages and disadvantages. The conventional transcranial approach can enable a direct access and sufficient exposure to the superior wall of the optic canal, which could help achieve an effective decompression of the optic nerve. However, the larger trauma from the craniotomy procedure may hinder the patients to accept the approach. The endoscopic transnasal approach has an advantage of minimal invasion, but the narrow surgical corridor and potential severe bleeding reduce the popularity of the approach.

For the supraorbital keyhole operation, a small incision located in the unilateral eyebrow is made and the sellar region can be reached through the intracranial natural space. Enlightened by the keyhole idea and with the aim to decrease the corresponding invasion of the conventional approaches, we tried to design a novel endoscopic approach to achieve an 
effective decompression of the optic nerve through the supraorbital keyhole approach. In this article, we describe the relevant anatomy of the modified transcranial approach and evaluate its feasibility.

\section{MATERIAL and METHODS}

This study was performed on 10 formalin-fixed adult human head specimens (20 hemispheres), whose deaths were not caused by cerebral diseases. All procedures were performed in the anatomy laboratory of Yangzhou University without any conflict of ethics. The laboratory equipment included endoscopes (Storz®, $4 \mathrm{~mm}$ in diameter and with 0- and 30-degree lenses), microsurgical instruments, a head-holder, an electric neurosurgical drill, a digital camera, a vernier caliper, and a video system.

Every head specimen was fixed in the head-holder. An endoscopic extradural approach for optic nerve decompression via the supraorbital keyhole was performed, and the correlative anatomical structures were observed. After that, the following measurements were recorded: the distance from the zygomatic process of the frontal bone (the key point) to the posterior foramen of the optic canal (PFOC), the adjacent relationship between the ophthalmic artery and the optic nerve, and the distance from the upper midpoint of the PFOC to the internal carotid artery. The data were summarized by the mean \pm SD.

\section{RESULTS}

Every head specimen was fixed in the head-holder and positioned with $15^{\circ}$ extension and $20-40^{\circ}$ of lateralization contra-lateral to the side of the approach. A $40 \mathrm{~mm}$ skin incision was created laterally from the supraorbital notch along the orbital rim within the eyebrow. The scalp and the frontal muscle were distracted. A single hole was bored near the zygomatic process of the frontal bone using a high-speed drill at the level of the frontal base. Afterwards, a small bone flap, 20-25 mm in diameter, was created and removed (Figure 1). The dura mater was bluntly peeled from the anterior cranial base using a dissector, and the endoscope (4 $\mathrm{mm}$; 0-degree) was introduced into the extradural space. Under the guidance of the dissector, the endoscope was advanced posteriorly and laterally until the sphenoid ridge appeared (Figures 2A, B). The endoscope was advanced deeply along the sphenoid ridge until the base of the anterior clinoid process (ACP) could be identified (Figure 2C). Under the 30-degree endoscope, the PFOC and the falciform ligament enveloping the optic nerve could be observed about $10 \mathrm{~mm}$ medially to the lateral edge of the ACP (Figure 2D). After the optic canal and its bone edge were identified, the roof and lateral wall of the optic canal were removed gradually using a diamond drill bit and a dissector to achieve $180^{\circ}$ bone decompression of the optic canal (Figures 3A, B). Furthermore, the following anatomical structures were observed: the optic nerve enveloped with the sheath, the sheath artery and the optic ring. More sufficient decompression could be achieved by further incising the falciform ligament and the optic sheath.
The optic nerve and the surrounding structures were further observed and measured in the cadaveric heads after removing the cerebral hemispheres (Figures 4, 5). After measurement, the distance from the key point to the optic canal was $59.32 \pm 2.27 \mathrm{~mm}$, and the distance from the upper midpoint of the PFOC to the internal carotid artery was $3.80 \pm 0.93 \mathrm{~mm}$.

In this study, the ophthalmic artery was identified inferiorlaterally to the optic nerve at the posterior foramen in $75 \%$ of the specimens and inferiorly in $25 \%$. While at the anterior foramen, the statistical results were as follows: inferior-laterally in $65 \%$, laterally in $15 \%$ and inferiorly in $20 \%$.

\section{DISCUSSION}

The most vulnerable part of the optic nerve to blunt head trauma is the intra-canalicular segment, because it lacks adequate space to relieve pressure (9). As a result, the optic neuropathy following accidental trauma usually results from the injury of the optic canal. The optic nerve injury, posttraumatic edema and further neurological damage will become a vicious spiral, which could lead to a serious consequence of blindness (19). A timely and efficient optic nerve decompression surgery can prevent the course and make it more possible to save sight. Even though preoperative blindness by an indirect optic nerve injury has a poor prognosis, some patients have a chance to recover when a wide decompression of the optic nerve is used (22). An improvement in visual acuity was achieved, although very limited in some cases, when surgery was performed as close as possible to the traumatic event (7). The traditional transcranial approach can support a sufficient space for the decompression of the optic nerve and especially is suitable for the cases combined with the intracranial hematoma near the anterior cranial base. However, this approach is not easily accepted because of its high invasion. With the development of minimally invasive surgical techniques, several novel approaches have been developed, including the transorbital, transantral, extranasal transethmoidal, and intranasal approaches (8), which have advantages such as the hidden incision, low invasion and less mental pressure of the

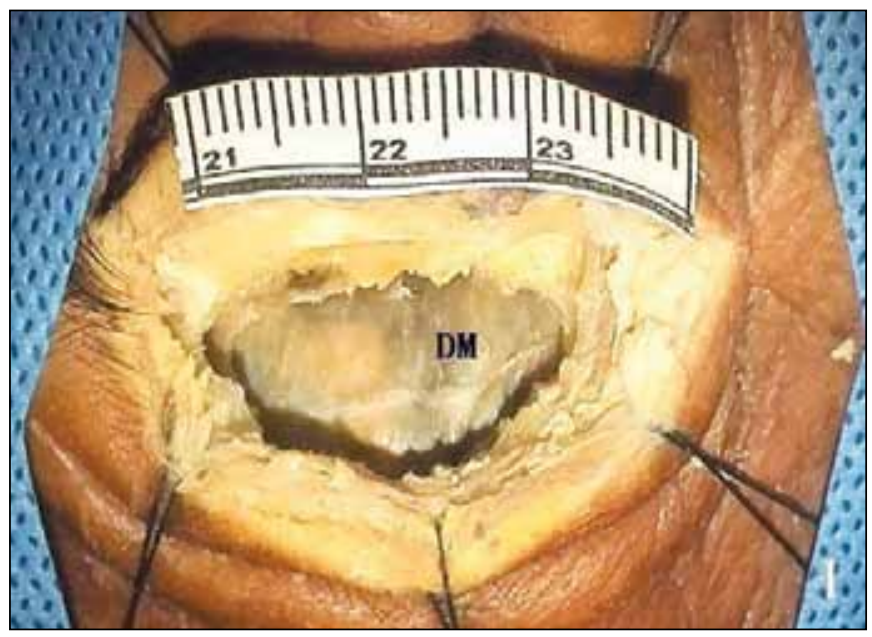

Figure 1: A small bone flap, 20-25 mm in diameter, was removed and the dura mater (DM) was exposed. 
patients. On the other hand, there are some disadvantages which effect their popularity: 1) the narrow surgical corridor and anatomic variations could effect the process and outcome of the optic nerve decompression (9); 2) The complications, including cerebrospinal fluid leakage and intracranial infection, more likely occur in the procedure via nasal sinus; 3) Due to the intimate relationship between arteries and optic nerve on its inferior surface, one must be careful to avoid the injury of the internal carotid artery or ophthalmic artery, which would lead to a serious consequence if happened (13). So, these non-transcranial surgical approaches have been chosen, in selected cases, to treat the traumatic optic neuropathy (10).
As the typical representatives of minimally invasive neurosurgical technique, the keyhole and the endoscopic surgeries have been used widely, especially in skull base neurosurgery $(2-6,11,12,20)$. The supraorbital keyhole approach is a classical keyhole approach, which has been commonly used to treat a host of disorders such as aneurysms of the anterior circulation, anterior fossa and sellar tumors, traumatic frontal lobe hematoma, etc. (16). Theoretically, the supraorbital keyhole approach could also be feasible for the decompression of the optic nerve, with the premise of achieving clear view of the PFOC and its surrounding structures.
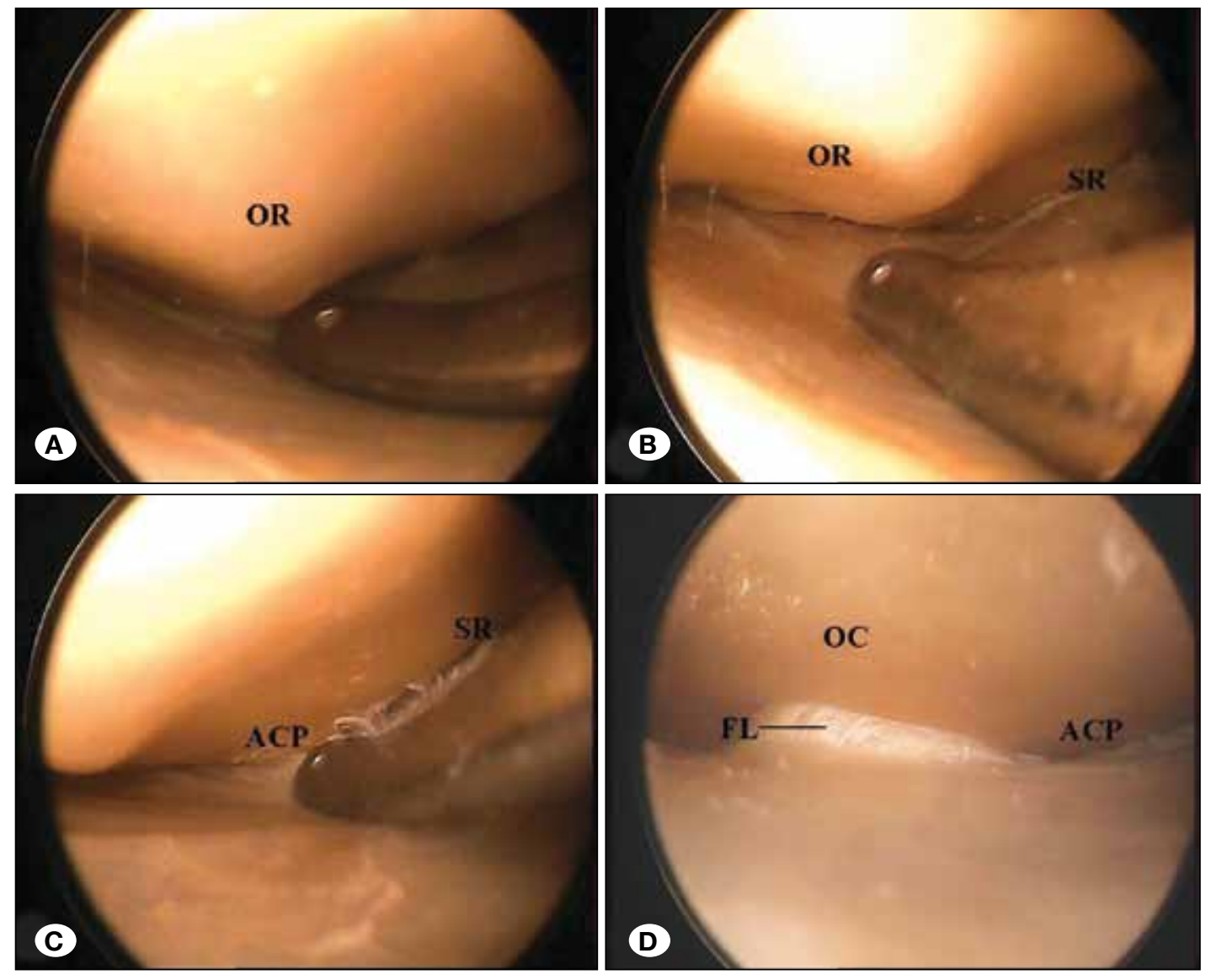

Figure 2: Via the supraorbital keyhole, an endoscope $(4 \mathrm{~mm}$; 0 -degree) was introduced into the extradural space and the dura mater was bluntly peeled. A) The bone eminence in the orbital roof (OR) was first observed. B) After the extradural apace was further explored using a dissector, the endoscope was advanced posteriorly and laterally until the sphenoid ridge (SR) appeared. C) Along the SR, the base of the anterior clinoid process (ACP) was be identified which could be used as a landmark; D) The roof of the optic canal (OC), the posterior foramen of the optic canal (PFOC) and the falciform ligament (FL) enveloping the optic nerve could be observed about $10 \mathrm{~mm}$ medially to the lateral edge of the ACP using a 30-degree endoscope.
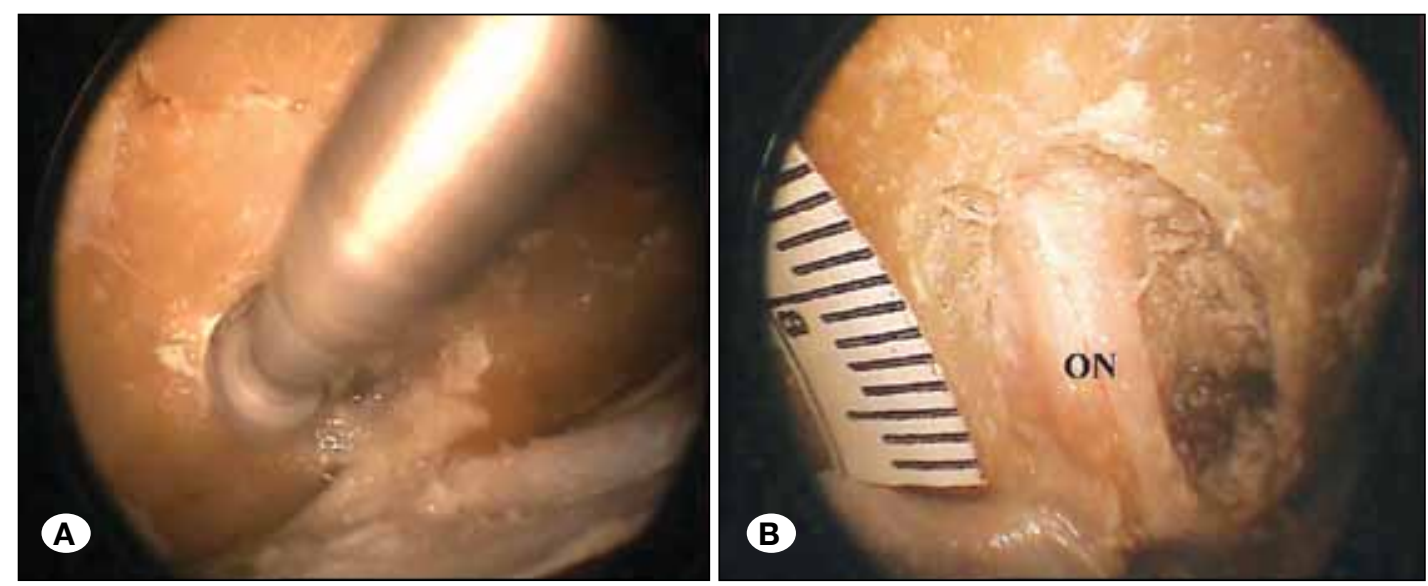

Figure 3: Under the 30-degree endoscope, the roof and lateral wall (the medial section of the anterior clinoid process) of the optic canal were removed gradually using the neurosurgical drill to achieve a sufficient decompression of the optic nerve (ON). 

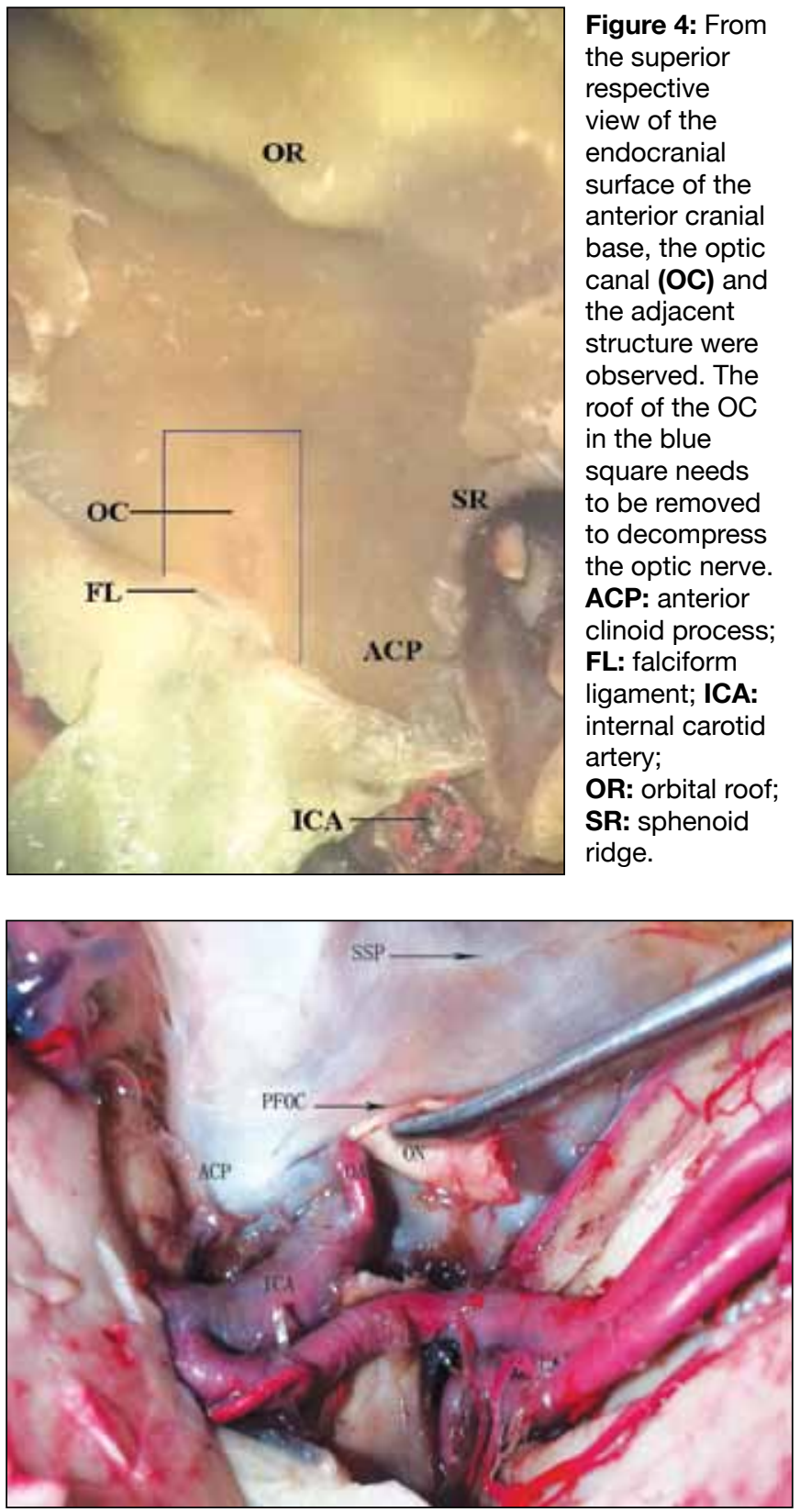

Figure 5: The adjacent relationship between the optic nerve and its surrounding arteries was observed in a cadaveric head. The ophthalmic artery (OA) from the internal carotid artery (ICA) entered the optic canal inferior-laterally to the optic nerve (ON). ACP: anterior clinoid process; PFOC: posterior foramen of the optic canal; SSP: suturae sphenoorbitalis.

Since their introduction, endoscopic techniques have advanced considerably beyond their initial usage in obstructive hydrocephalus (17). Compared with the microscope, the endoscope has its own advantages, including the minimally invasive corridor, deep illumination and more angles of view, which permit the surgeon to see into spaces outside the line of site available with traditional microsurgical techniques, to look around surgical corners, and improve illumination and magnification in deep cavities. The combination of the keyhole concept and the endoscopic technique can make it possible to perform minimally invasive and safe optic nerve decompression (1).

Structures including the orbital roof, the SR, the ACP, and the PFOC could be exposed and identified successively under the endoscope via the supraorbital keyhole approach. In the present approach, the SR and the ACP were important landmarks to find and identify the PFOC. It is not indispensable to drill the bone eminences of the anterior cranial base to expose the PFOC because of the deep illumination and multiangle view of the endoscope, which means less trauma and shorter operation time. Extreme care was taken to remove the roof and lateral wall of the optic canal using a drill. A thin shell of bone, which is later fractured and elevated with a dissector, should be left on the superior aspect of the nerve. According to the adjacent relationship between the optic nerve and its surrounding arteries, it is relatively less possible to trigger severe bleeding. However, it should be noted that the over removal of the ACP may increase the risk of the injury to the ophthalmic artery and the internal carotid artery. Further decompression of the optic nerve could be achieved by incising the falciform ligament and optic sheath parallel to the long axis of the optic nerve. While incising, care was taken to not injure the sheath artery which nourishes the optic nerve and may be observed through the endoscope. In addition, the potential occurrence of cerebrospinal fluid leakage should be considered after incising the optic sheath.

The theoretical advantages of the present approach are as follows: 1) the procedure is performed via a $40 \mathrm{~mm}$ incision in the eyebrow and a small bone flap, which is more easily accepted by patients because of the smaller effect on the appearance; 2) The application of the micro bone hole and the extradural procedure can avoid invalid exposure of the brain tissue, restrict excessive stretch to the brain, and theoretically reduce the occurrence rate of many complications such as intracranial infection and iatrogenic brain injury; 3) Owing to the advantages of deep illumination and more angles of view, the usage of the endoscope can simplify the procedure of drilling the skull, and can safely and effectively decompress the optic nerve with a clear vision.

In cases with a large frontal sinus, attention should be paid to timely closing the opened frontal sinus to avoid related infection. The main disadvantage of this approach is that it may be unsuitable for the optic neuropathy caused by fracture of the medial or inferior wall of the optic canal. As this is a cadaveric study, clinical studies are needed to demonstrate the safety and efficacy of this approach. The exquisite endoscopic technique is the premise to clinically performing this surgical approach.

\section{CONCLUSION}

According to this cadaveric study, minimally invasive optic nerve decompression could be achieved with clear vision using the endoscopic supraorbital keyhole approach. This method could be a surgical option for traumatic optic neuropathy combined with optic canal stenosis. The clinical efficacy needs to be studied further. 


\section{- REFERENCES}

1. Beer-Furlan A, Evins Al, Rigante L, Burrell JC, Anichini G, Stieg $P E$, Bernardo A: Endoscopic extradural anterior clinoidectomy and optic nerve decompression through a pterional port. $\mathrm{J}$ Clin Neurosci 21: 836-840, 2014

2. Cappabianca P, Cavallo LM, Esposito F, De Divitiis O, Messina A, de Divitiis E: Extended endoscopic endonasal approach to the midline skull base: The evolving role of transsphenoidal surgery. Adv Tech Stand Neurosurg 33:151-199, 2008

3. Cavallo LM, Cappabianca P, Messina A, Esposito F, Stella L, de Divitiis E, Tschabitscher M: The extended endoscopic endonasal approach to the clivus and cranio-vertebral junction: Anatomical study. Childs Nerv Syst 23:665-671, 2007

4. Cavallo LM, de Divitiis O, Aydin S, Messina A, Esposito F, laconetta G, Talat K, Cappabianca P, Tschabitscher M: Extended endoscopic endonasal transsphenoidal approach to the suprasellar area: Anatomic considerations-part 1. Neurosurgery 62:1202-1212, 2008

5. Cavallo LM, Messina A, Cappabianca P, Esposito F, de Divitiis E, Gardner P, Tschabitscher M: Endoscopic endonasal surgery of the midline skull base: Anatomical study and clinical considerations. Neurosurg Focus 19:E2, 2005

6. Colpan ME, Sekerci Z, Cakmakci E, Donmez T, Oral N, Mogul DJ: Virtual endoscope-assisted intraeranial aneurysm surgery: Evaluation of fifty-eight surgical cases. Minim Invasive Neurosurg 50:27-32, 2007

7. Emanuelli E, Bignami M, Digilio E, Fusetti S, Volo T, Castelnuovo P: Post-traumatic optic neuropathy: Our surgical and medical protocol. Eur Arch Otorhinolaryngol 272(11):3301-3309, 2015

8. Hart CK, Theodosopoulos PV, Zimmer LA: Anatomy of the optic canal: A computed tomography study of endoscopic nerve decompression. Ann Otol Rhinol Laryngol 118:839-844, 2009

9. Hathiram BT, Khattar VS, Sonawane HP, Watve PJ: Traumatic optic neuropathy-our experience. Indian J Otolaryngol Head Neck Surg 62:229-235, 2010

10. Horiguchi K, Murai H, Hasegawa Y, Mine S, Yamakami I, Saeki N: Endoscopic endonasal trans-sphenoidal optic nerve decompression for traumatic optic neuropathy-technical note. Neurol Med Chir 50:518-522, 2010
11. Komatsu F, Komatsu M, Di leva A, Tschabitscher M: Endoscopic extradural subtemporal approach to lateral and central skull base: A cadaveric study. World Neurosurg 80:591-597, 2012

12. Lan Q, Gong Z, Kang D, Zhang H, Qian Z, Chen J, Huang $Q$ : Microsurgical experience with keyhole operations on intracranial aneurysms. Surg Neurol 66: S2-9, 2006

13. Li HB, Shi JB, Cheng L, Yun O, Xu G: Salvage optic nerve decompression for traumatic blindness under nasal endoscopy: Risk and benefit analysis. Clin Otolaryngol 32:447-251, 2007

14. Li J, Wang J, Jing X, Zhang W, Zhang X, Qiu Y: Transsphenoidal optic nerve decompression: An endoscopic anatomic study. J Craniofac Surg 19:1670-1674, 2008

15. Locatelli M, Caroli M, Pluderi M, Motta F, Gaini SM, Tschabitscher M, Scarone P: Endoscopic transsphenoidal optic nerve decompression: An anatomical study. Surg Radiol Anat 33:257-262, 2011

16. Ma Y, Lan Q: Supraorbital keyhole approach to upper basilar artery aneurysms via the optico-carotid window: A cadaveric anatomic study and preliminary application. Minim Invasive Neurosurg 54:228-235, 2011

17. Mohanty A, Thompson BJ, Patterson J: Initial experience with endoscopic side cutting aspiration system in pure neuroendoscopic excision of large intraventricular tumors. World Neurosurg 80:655.e15-21, 2013

18. Peng A, Li Y, Hu P, Wang Q: Endoscopic optic nerve decompression for traumatic optic neuropathy in children. Int J Pediatr Otorhinolaryngol 75:992-998, 2011

19. Steinsapir KD, Goldberg RA: Traumatic optic neuropathy: A critical update. Compr Ophthalmol Update 6:11-21, 2005

20. Sun JQ, Han DM, Li YX, Gong SS, Zan HR, Wang T: Combined endoscope-assisted translabyrinthine subtemporal keyhole approach for vestibular schwannoma and auditory midbrain implantation: Cadaveric study. Acta Otolaryngol 130:11251129, 2010

21. Yang QT, Zhang GH, Liu X, Ye J, Li Y: The therapeutic efficacy of endoscopic optic nerve decompression and its effects on the prognoses of 96 cases of traumatic optic neuropathy. $\mathrm{J}$ Trauma Acute Care Surg 72:1350-1355, 2012

22. Zhilin G, Huoniu O, Zhihua C, Guorong D: Wide optic nerve canal decompression for the treatment of blindness resulting from an indirect optic nerve injury. J Craniofac Surg 22:14631465, 2011 